Retrospective Clinical Study

Volume 1 - Issue 1

\title{
Periodontitis Risk in Patients With and Without Systemic Lupus Erythematosus: A Retrospective Study
}

\author{
James Y. W. Wu ${ }^{1}$, Vijaya Lakshmi Pavani Molli ${ }^{2}$, Chinhua Hsiao ${ }^{3, *}$ \\ ${ }^{1}$ Private Practice, San Antonio, Texas \\ ${ }^{2}$ Private Practice, Dallas, Texas \\ ${ }^{3}$ Assistant Professor, Graduate Periodontology and Implantology Resident at Temple University Kornberg School of Dentistry, \\ Philadelphia, Pennsylvania
}

*Corresponding author: Chinhua Hsiao, Assistant Professor, Graduate Periodontology and Implantology Resident at Temple University Kornberg School of Dentistry, Philadelphia, Pennsylvania

Received date: 15 June, 2021 | Accepted date: 6 August, 2021 | Published date: 13 August, 2021

Citation: Wu J.Y.W, Molli VLP, Hsiao C (2021) Periodontitis Risk in Patients With and Without Systemic Lupus Erythematosus: A Retrospective Study. J Dent Oral Epidemiol 1(1). doi https://doi.org/10.54289/JDOE2100104

Copyright: () $2021 \mathrm{Wu}$ J.Y.W, et al. This is an open-access article distributed under the terms of the Creative Commons Attribution License, which permits unrestricted use, distribution, and reproduction in any medium, provided the original author and source are credited.

\begin{abstract}
Objectives: Systemic lupus erythematosus is a systemic, long-term autoimmune condition that has chronic inflammatory effects in connective tissue throughout the body. There are numerous studies that have examined the association between systemic lupus erythematosus and chronic periodontitis, with varying conclusions. The purpose of this cross-sectional study is to evaluate and compare the risk for periodontitis in patients with SLE to patients without SLE.

Materials and Methods: Medical and dental records were retrospectively reviewed for patients that had been admitted to the Temple University School of Dentistry from 2010 to 2018. A roster of 22 SLE positive patients were generated from the Temple University patient database and matched to a control population of 22 patients without SLE. Periodontal probing depths were then documented and used to evaluate periodontal statuses in both test and control groups. Sites with probing depths $\geq 5 \mathrm{~mm}$ were considered to be at increased risk for periodontal breakdown. Prevalence was defined as the percentage of individuals having at least one site with a $\geq 5 \mathrm{~mm}$ probing depth, and extent was defined as the average percentage of sites with increased periodontitis risk. The number of missing teeth in patients from each group were also recorded as a secondary outcome.

Results: The prevalence of $\geq 5 \mathrm{~mm}$ probing depths in SLE and control groups was 50\% (10/20) and 40.9\% (9/22), respectively. Calculations of relative risk (1.22) and odds ratio (1.44) were not statistically significant between the two populations ( $\mathrm{p}>0.05$ ). The extent of $\geq 5 \mathrm{~mm}$ probing depths was $1.5 \%$ in SLE patients and $3.7 \%$ in healthy patients, which was also not significant between groups ( $\mathrm{p}>0.05)$. SLE patients were missing an average of 9.6 teeth per individual compared to 3.8 in healthy patients $(\mathrm{p}<0.05)$.
\end{abstract}


Conclusions: The results of the present study indicate that patients with SLE do not have an increased risk for periodontitis when compared to patients without SLE. Risk analysis on the prevalence and the extent of deeper probing depths were not statistically different between SLE and control groups. Further studies with a larger sample size and elimination of unseen confounders are needed in order to validate our results.

An interesting observation was the finding that SLE patients have a significantly greater number of missing teeth. The exact mechanism through which SLE patients experience periodontal breakdown and increased tooth loss is an avenue that warrants future research.

Keywords: systemic lupus erythematosus (SLE); chronic periodontitis

Abbreviations: SLE: Systemic Lupus Erythematosus, CP: Chronic Periodontitis, SLEDAI: Systemic Lupus Erythematosus Disease Activity Index, BOP: Bleeding on Probing, CAL: Clinical Attachment Level.

\section{Introduction}

Systemic Lupus Erythematosus (SLE), also called lupus, is a chronic autoimmune disease that has clinical manifestations throughout the human body. The condition results in widespread inflammation and can affect multiple organ systems, including the heart, kidneys, joints, and blood vessels. Although detection methods have improved in recent years, diagnosing the disease is challenging and often requires an array of x-rays, laboratory tests, and clinical evaluations. Symptoms can be mild to more severe and may include oral ulcers, joint pain, chest pain, fatigue, weight loss, hair loss, photosensitivity, and in about half of SLE patients, a characteristic "butterfly rash" [1].

There are about 20 to 70 cases of SLE per 100,000 individuals in the United States, with a greater prevalence in individuals of Black, Asian or Hispanic descent. It is also found nine times more frequently in women than in men. Studies have demonstrated that there may be a hormonal mechanism that leads to the increased prevalence of SLE in females and that the immunological genes that contribute to SLE vulnerability are located on the X-chromosome. Although the exact etiology is not widely known, the onset of lupus can be influenced strongly by both genetic predisposition and environmental triggers [2].

Periodontitis is another multi-factorial, chronic inflammatory disease that affects the supporting structures of the teeth, such as cementum, bone, and periodontal ligament. As dental plaque and its associated microorganisms accumulate around the gingival margin, they can elicit an amplified host immune response that leads to bone loss around the teeth. The resulting damage often leads to deep pocketing, gingival recession, tooth mobility, and tooth loss. It is estimated that periodontitis is prevalent in $20-50 \%$ of the world's population, making it the most common condition affecting the oral cavity [3].

While pathogenic bacteria have been implicated as the principal etiological agent of periodontitis, studies demonstrate that there is a strong genetic contribution towards susceptibility to the disease. Much like SLE, host systemic and local factors interact with environmental triggers to modulate the immune response and influence progression of periodontal breakdown [4].

There are abundant studies in the literature supporting the idea that periodontal disease does not affect just the oral cavity but has significant involvement with systemic conditions like diabetes and cardiovascular disease [3]. Another such relationship is the potential association between chronic periodontitis and SLE. A study conducted by Rhodus [5] examined the oral manifestations of SLE and found that over $90 \%$ of patients with SLE had periodontitis, significantly higher than the global average. Another study by Aurlene [6] found a positive association between the SLE Disease Activity Index (SLEDAI), which is used to quantify SLE disease activity in clinical research, and severity of 
periodontitis [7]. examined the effects of non-surgical periodontal therapy on the SLEDAI in SLE patients undergoing immunosuppressive therapy. Results at the end of his study demonstrated that along with a significant improvement in clinical parameters after periodontal therapy, there was an analogous reduction in the SLEDAI.

Perhaps this bi-directional relationship can be attributed to the shared pro-inflammatory profile between SLE and periodontitis. IL-1 $\beta$ and TNF- $\alpha$, inflammatory cytokines that are typically elevated in periodontitis patients, were found to be abundant in SLE patients [8] Even more surprising was the correlation that in SLE patients, shifts in oral microbiota towards known periodontal pathogens like $T$. denticola and $T$. forsythia led to increased SLE activity and severity [9].

Nevertheless, case-controls by other authors have found different, even contradictory results. Meyer et al [10] found periodontal parameters to be statistically similar between SLE and non-SLE patients, while research by Mutlu et al [11] actually found better periodontal parameters in patients with SLE. Literature on the association between these two conditions had shown that Chronic Periodontitis occurred early in SLE patients [12].

The aim of this study is to compare periodontal probing depths in patients with SLE to patients without SLE, and by doing so, provide additional insight into whether SLE patients have an elevated risk for periodontal breakdown. The null hypothesis was that SLE patients do not have any significant difference in terms of prevalence and extent of probing depths $\geq 5 \mathrm{~mm}$.

\section{Materials And Methods}

The present investigation is an observational study of crosssectional, retrospective design conducted at the Temple University School of Dentistry. Research protocols were reviewed and approved by the Temple University Office for Human Subjects Protections Institutional Review Board. Patient data was obtained through the Axium database. Subject inclusion criteria were patients that were at least 20 years of age and had been admitted to Temple University between 2010 to 2018. Subjects were excluded if they had a history of uncontrolled diabetes, were current smokers, required antibiotic prophylaxis prior to dental appointments, or had undergone periodontal therapy within the past six months. The test group included only patients that were diagnosed with Systemic Lupus Erythematosus, while the control group included patients that did not have a history of the condition.

An automated search of the existing Temple University patient population yielded a roster of 39 SLE patients. Diagnoses of SLE were based on self-reports by the patient and by letters provided by the patients' physicians. Patients with drug-induced lupus or cutaneous lupus erythematosus were not included in the study.

Application of the inclusion and exclusion criteria left a roster of eligible SLE patients. To eliminate potential confounding factors, the same number of control patients without SLE were then selected at random from the Temple University patient database and sorted specifically to match the test group in terms of gender and decade of birth. This resulted in analogous pairs of SLE and healthy control patients that shared the same gender and age bracket.

All clinical periodontal evaluations had been performed at Temple University by third- and fourth-year dental students, under the supervision of Board-certified Periodontists and recorded into Axium dental software. Probing depths were measured at six sites for each tooth (mesial-buccal, midbuccal, distal-buccal, distal-lingual, mid-lingual, and mesiallingual). Third molars were excluded from the analysis, for a total of 168 sites in patients with a fully intact dentition. Of the 44 total patients in the study, 2 patients from the SLE group were completely edentulous. Information on gingival recession, clinical attachment level, bleeding on probing, mobility, and width of keratinized tissue were recorded in several, but not all patients.

Limited available periodontal parameters were recorded for all test and control subjects. Review of patient data revealed that documentation of clinical periodontal parameters was not always comprehensive and information on gingival recession, clinical attachment level, or BOP were left vacant for many subjects. Periodontal probing depth was the only consistently documented parameters for all subjects. As a result, probing depth was the exclusive clinical parameter used to establish 
the burden and potential risk of periodontitis for each patient. Sites that were considered risk factors for future periodontal breakdown and tooth loss were any probing depths that were $\geq 5 \mathrm{~mm}$.

Patients that had incomplete periodontal probing records were excluded from the present study. Probing depths were the primary outcome. Patients were classified as having an increased risk for periodontitis if they possessed one or more sites with probing depth $\geq 5 \mathrm{~mm}$, irrespective of overall probing depth severity. On the other hand, subjects that had no probing depths $\geq 5 \mathrm{~mm}$ were classified as nominal risk. Prevalence was defined as the percentage of subjects having at least one tooth with $\geq 5 \mathrm{~mm}$ probing depths, while extent was defined as the percentage of $\geq 5 \mathrm{~mm}$ sites among total sites remaining, per person. Our secondary outcome was the number of missing teeth, discounting the third molars. The quantity and type (anterior, premolar, molar) of missing teeth from every individual were recorded for the test and control groups.

Statistical assessments were performed using the Statistical Package for Social Sciences software. The Mann-Whitney U test was used to for comparative analysis between patients with SLE and healthy control groups. Within the SLE and healthy control groups, associations between age and periodontal parameters were analyzed using the KruskalWallis test. P-values of $<0.05$ were considered to be significant.

\section{Results}

Initially, thirty-nine patients with a diagnosis of SLE were found in the patient database at Temple University.
Of these 39 patients, 17 were removed either due to application of our exclusion criteria, or because they did not have complete documentation of probing depths and missing teeth. Twenty-two SLE patients were left that met the eligibility criteria, of which $100 \%$ were female. After selection of the matched control individuals, a total of fortyfour patients were enrolled in the study, comprised of 22 SLE and 22 healthy control patients. The mean age was 51.7 years for the SLE group and 49.9 years for the control group. Information on patient race or socioeconomic statuses were not available in the patient records.

Among the 22 patients with SLE, 10 were positive for probing depths $\geq 5 \mathrm{~mm}$. Two patients were excluded from the analysis because they were completely edentulous and thus had no sites for periodontal breakdown. The remaining 10 patients had probing depths of 4 or less. Of the healthy control patients, 9 had probing depths $\geq 5 \mathrm{~mm}$, while the remaining 13 patients did not. The prevalence of $\geq 5 \mathrm{~mm}$ probing depths in tests and control groups was 50\% (10/20) and 40.9\% $(9 / 22)$, respectively. Statistical analysis showed the risk of having at least one site with probing depths $\geq 5 \mathrm{~mm}$ in SLE patients to be $22 \%$ greater $(R R=1.22)$ when compared to the control population without SLE, though this finding was not statistically significant ( $p>0.05$ ) (Table 1). The odds ratio suggested a greater association between increased probing depths and SLE $(\mathrm{OR}=1.44)$, which was also found to not be statistically significant $(p>0.05)$ (Table 2).

\begin{tabular}{|l|l|}
\hline Relative risk & 1.2222 \\
\hline $95 \% \mathrm{Cl}$ & 0.6276 to 2.3803 \\
\hline z statistic & 0.590 \\
\hline Significance level & $\mathrm{P}=0.5551$ \\
\hline NNT (Harm) & 11.000 \\
\hline $95 \% \mathrm{Cl}$ & 2.557 (Harm) to $\infty$ to 4.779 (Benefit) \\
\hline
\end{tabular}

Table 1. Relative Risk of $\geq 5 \mathrm{~mm}$ probing depths in SLE vs Healthy patients 


\begin{tabular}{|l|l|}
\hline Odds ratio & 1.4444 \\
\hline $95 \% \mathrm{Cl}:$ & 0.4260 to 4.8972 \\
\hline z statistic & 0.590 \\
\hline Significance level & $\mathrm{P}=0.5550$ \\
\hline
\end{tabular}

Table 2. Odds ratio of $\geq 5 \mathrm{~mm}$ probing depths in SLE vs non-SLE patients

\begin{tabular}{|l|r|r|r|}
\hline & $\begin{array}{l}\text { SLE } \\
\text { PATIENTS }\end{array}$ & $\begin{array}{l}\text { HEALTHY } \\
\text { PATIENTS }\end{array}$ & P-value \\
\hline $\begin{array}{l}\text { Average \% of } \geq 5 \mathrm{~mm} \\
\text { Pockets (Extent) }\end{array}$ & $1.5 \%$ & $3.7 \%$ & 0.968 \\
\hline $\begin{array}{l}\text { Average Number of Missing } \\
\text { Teeth }\end{array}$ & 9.6 & 3.8 & 0.017 \\
\hline
\end{tabular}

Table 3. Mean clinical parameters in SLE and Healthy patients

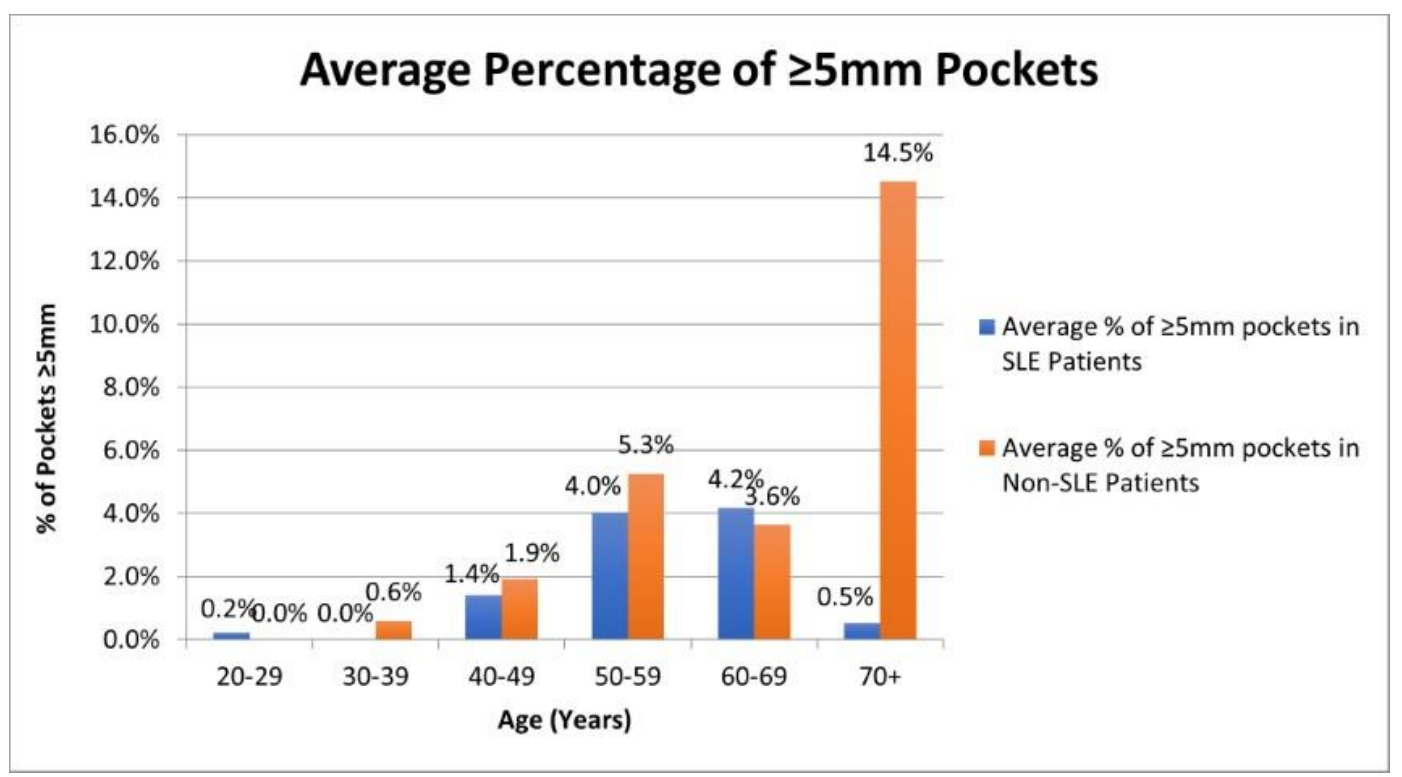

Figure 1. Average Percentage of $\geq 5 \mathrm{~mm}$ Pockets in SLE and Non-SLE patients

Patients from the SLE and control groups were missing an average of 9.6 and 3.8 teeth, respectively. Statistically, the average number of missing teeth was found to be significantly greater in SLE patients versus healthy control groups $(p<0.05)$. There was also a statistically significant association between the number of missing teeth and increased patient age. Table 3 depicts the average number of missing teeth in both test and control groups. The extent, or percentage of pockets $\geq 5 \mathrm{~mm}$ among total sites was, on average, $1.5 \%$ in the SLE group, while the average percentile of $\geq 5 \mathrm{~mm}$ pockets for the healthy group was slightly higher at $3.70 \%$ (Table 3).

The progression of probing depths $\geq 5 \mathrm{~mm}$ in patients of increasing age can be seen graphically in Figure 1. The extent of $\geq 5 \mathrm{~mm}$ probing depths, however, were not found to be statistically significant between test and control groups, or between age groups.

Figure 2 contains a side-by-side comparison of number of 
missing teeth in SLE and healthy control groups and is separated into the type of missing teeth. The most commonly missing teeth in both test and control groups were molars, followed by premolars, and lastly, anterior teeth (incisors and canines).
The comparative statistical analysis used to compare the SLE, and control groups can be seen in Tables 4, 5, 6, and 7 . Clinical parameters were processed both as raw numbers and as percentages, with the same results. No significant differences in terms of probing depths were found between age groups or SLE status.

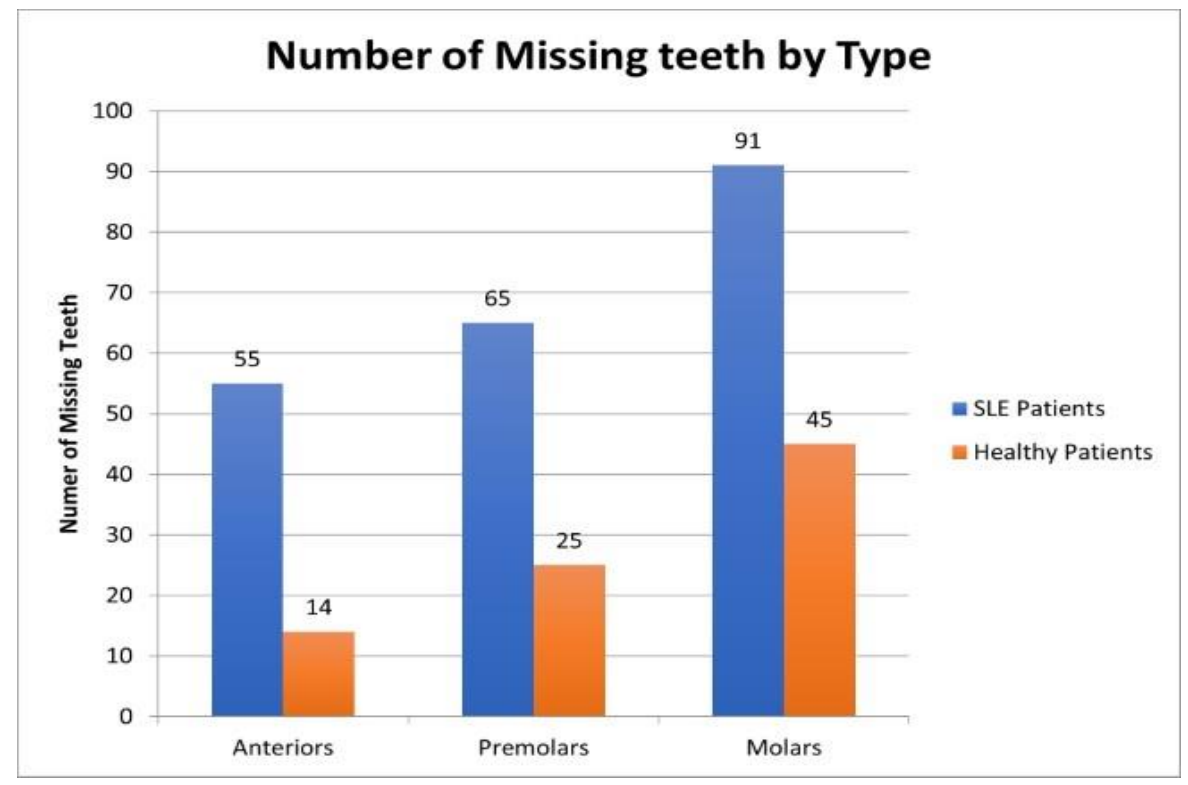

Figure 2. Number and type of missing teeth in SLE and Healthy controls

\begin{tabular}{|c|c|c|c|}
\hline & \multicolumn{3}{|c|}{ Ranks } \\
\hline & SLE & $N$ & Mean Rank \\
\hline \multirow[t]{3}{*}{ Missing Teeth } & 0 & 22 & 17.93 \\
\hline & 1 & 22 & 27.07 \\
\hline & Total & 44 & \\
\hline \multirow[t]{3}{*}{$4 \mathrm{~mm}$ Pockets } & 0 & 22 & 21.55 \\
\hline & 1 & 22 & 23.45 \\
\hline & Total & 44 & \\
\hline \multirow[t]{3}{*}{$5 \mathrm{~mm}$ Pockets } & 0 & 22 & 21.93 \\
\hline & 1 & 21 & 22.07 \\
\hline & Total & 43 & \\
\hline
\end{tabular}

\begin{tabular}{l|r|r|r}
\multicolumn{3}{c}{ Test Statistics $^{\mathbf{a}}$} & \\
& Missing Teeth & 4mm Pockets & 5mm Pockets \\
\hline Asymp. Sig. (2-tailed) & .017 & .615 & .968 \\
\hline
\end{tabular}

Table 4. Mann Whitney Test (Numerical data) comparing SLE vs Non-SLE 


\begin{tabular}{|c|c|c|c|c|c|}
\hline \multicolumn{6}{|c|}{ Ranks } \\
\hline & SLE & $N$ & Meal & Rank & \\
\hline \multirow[t]{3}{*}{ Missing Teeth } & 0 & 22 & & 17.93 & \\
\hline & 1 & 22 & & 27.07 & \\
\hline & Total & 44 & & & \\
\hline \multirow[t]{3}{*}{ Percent_4mn } & 0 & 22 & & 20.73 & \\
\hline & 1 & 22 & & 24.27 & \\
\hline & Total & 44 & & & \\
\hline \multirow[t]{5}{*}{ Percent_5mm } & 0 & 22 & & 22.36 & \\
\hline & 1 & 22 & & 22.64 & \\
\hline & Total & 44 & & & \\
\hline & \multicolumn{5}{|c|}{ Test Statistics $^{a}$} \\
\hline & Missing Teeth & \multicolumn{2}{|c|}{$\begin{array}{c}\text { Percent_4m } \\
\mathrm{m}\end{array}$} & Perc & $n t+5 m$ \\
\hline ig. (2-tailed) & .017 & & .349 & & .938 \\
\hline
\end{tabular}

Table 5. Mann Whitney Test (Percentages) comparing SLE vs Non-SLE

\section{Ranks}

\begin{tabular}{|c|c|c|c|}
\hline & Age_Cat & $\mathrm{N}$ & Mean Rank \\
\hline \multirow[t]{8}{*}{ Missing Teeth } & 2.00 & 6 & 9.50 \\
\hline & 3.00 & 4 & 15.00 \\
\hline & 4.00 & 12 & 22.21 \\
\hline & 5.00 & 8 & 25.94 \\
\hline & 6.00 & 8 & 24.13 \\
\hline & 7.00 & 4 & 34.50 \\
\hline & 8.00 & 2 & 34.00 \\
\hline & Total & 44 & \\
\hline \multirow[t]{8}{*}{$4 \mathrm{~mm}$ Pockets } & 2.00 & 6 & 16.50 \\
\hline & 3.00 & 4 & 11.13 \\
\hline & 4.00 & 12 & 21.79 \\
\hline & 5.00 & 8 & 29.63 \\
\hline & 6.00 & 8 & 22.69 \\
\hline & 7.00 & 4 & 29.38 \\
\hline & 8.00 & 2 & 24.50 \\
\hline & Total & 44 & \\
\hline \multirow[t]{8}{*}{$5 \mathrm{~mm}$ Pockets } & 2.00 & 6 & 14.83 \\
\hline & 3.00 & 3 & 18.17 \\
\hline & 4.00 & 12 & 23.46 \\
\hline & 5.00 & 8 & 23.50 \\
\hline & 6.00 & 8 & 24.94 \\
\hline & 7.00 & 4 & 23.63 \\
\hline & 8.00 & 2 & 19.50 \\
\hline & Total & 43 & \\
\hline
\end{tabular}




\section{Test Statistics ${ }^{a, b}$}

\begin{tabular}{l|r|r|r} 
& Missing Teeth & $4 \mathrm{~mm}$ Pockets & $5 \mathrm{~mm}$ Pockets \\
\hline Asymp. Sig. & .036 & .204 & .710 \\
\hline
\end{tabular}

a. Kruskal Wallis Test

b. Grouping Variable: Age_Cat

Table 6. Kruskal-Wallis Test (Numerical Data) by Age Category

\section{Ranks}

\begin{tabular}{|c|c|c|c|}
\hline & Age_Cat & $\mathrm{N}$ & Mean Rank \\
\hline \multirow[t]{8}{*}{ Missing Teeth } & 2.00 & 6 & 9.50 \\
\hline & 3.00 & 4 & 15.00 \\
\hline & 4.00 & 12 & 22.21 \\
\hline & 5.00 & 8 & 25.94 \\
\hline & 6.00 & 8 & 24.13 \\
\hline & 7.00 & 4 & 34.50 \\
\hline & 8.00 & 2 & 34.00 \\
\hline & Total & 44 & \\
\hline \multirow[t]{8}{*}{ Percent_4mm } & 2.00 & 6 & 15.50 \\
\hline & 3.00 & 4 & 10.63 \\
\hline & 4.00 & 12 & 20.92 \\
\hline & 5.00 & 8 & 30.44 \\
\hline & 6.00 & 8 & 22.31 \\
\hline & 7.00 & 4 & 32.50 \\
\hline & 8.00 & 2 & 25.75 \\
\hline & Total & 44 & \\
\hline \multirow[t]{8}{*}{ Percent_5mm } & 2.00 & 6 & 15.50 \\
\hline & 3.00 & 4 & 16.75 \\
\hline & 4.00 & 12 & 23.83 \\
\hline & 5.00 & 8 & 24.94 \\
\hline & 6.00 & 8 & 25.69 \\
\hline & 7.00 & 4 & 24.50 \\
\hline & 8.00 & 2 & 20.50 \\
\hline & Total & 44 & \\
\hline
\end{tabular}

\section{Test Statistics ${ }^{\mathrm{a}, \mathrm{b}}$}

\begin{tabular}{lr|r|r} 
& Missing Teeth & $\begin{array}{c}\text { Percent_4m } \\
\mathrm{m}\end{array}$ & $\begin{array}{c}\text { Percent_5m } \\
\mathrm{m}\end{array}$ \\
\hline Asymp. Sig. & .036 & .075 & .613 \\
\hline
\end{tabular}
a. Kruskal Wallis Test
b. Grouping Variable: Age_Cat

Table 7. Kruskal-Wallis Test (Percentages) by Age Category 


\section{Discussion}

The relationship between periodontal disease and systemic conditions are often very complex. The multi-factorial nature of periodontitis and SLE make understanding their association ever more challenging. Periodontitis is similar to SLE in that it can have genetic and systemic risk factors, but there are numerous local risk factors, as well.

One important risk factor of periodontitis progression is the presence of increased probing depths. Deep probing depths indicate a physiologic pocket that can harbor pathogenic microorganisms and dental calculus, leading to inflammation and alveolar bone destruction. Our study used probing depths $\geq 5 \mathrm{~mm}$ as a threshold to represent sites at greater risk for periodontal breakdown. This probing depth threshold was based after findings from a study by Matuliene et al [13], who found that pockets $\geq 5 \mathrm{~mm}$ represented a risk factor for progression of periodontitis and tooth loss. Waerhaug [14] determined that the chances of achieving complete plaque removal during non-surgical therapy from surfaces with $>5 \mathrm{~mm}$ probing depths was only $11 \%$. In their development of a functional diagram for periodontal risk assessment, Lang and Tonetti et al [15] also described the prevalence of $\geq 5 \mathrm{~mm}$ probing depths as a risk indicator for recurrent disease.

It is important to note that definitions of periodontitis have often varied in past studies that examined the association between SLE and periodontitis [12]. The current study did not attempt to diagnose periodontitis, but rather used probing depths to identify SLE and healthy patients that may have an increased risk for periodontal disease. Due to the chronic and rare nature of SLE, this proposal was appropriate when attempting to determine whether SLE patients were at greater risk for periodontal disease. Using the Prevalence (percentage of subjects) and extent (percentage of sites per subject) of deeper probing depths makes sense from a public health perspective- atypical conditions that may need more frequent maintenance visits or surveillance can be identified.

The analysis of periodontal probing depths in the present study indicate that compared to healthy patients, patients with SLE do not have a statistically increased risk of future periodontal breakdown. Any pockets greater than $5 \mathrm{~mm}$ were considered at greater risk for recurrent periodontitis, and while SLE patients did have a greater prevalence of $\geq 5 \mathrm{~mm}$ probing depths, this difference was not statistically significant from the healthy controls. Calculations of relative risk and odds ratio suggests that patients with SLE may have a slightly higher association with deeper probing depths. However, this study was unable to state that such an effect exists due to the high p-value.

Likewise, there were no statistically significant differences in the extent of periodontal $\geq 5 \mathrm{~mm}$ probing depths between the two cohorts. Although periodontal probing depth by itself does not represent a comprehensive assessment of the periodontal condition, increased probing depths can help assess elevated risk for periodontitis. The results obtained suggest that the presence of SLE does not have an effect on the extent of deeper probing depths that may be predictors of periodontal disease. Kobayashi [16] published similar results in 2007 when he found that periodontal parameters were similar in patients with SLE relative to those without it.

SLE is a relatively rare, multifaceted condition with different levels of systemic involvement and the number of SLE positive patients presenting to the Temple University dental clinic was very low. The small sample size is a significant limitation in the present study- statistical analysis was underpowered and because of the high p-values, a significant association could not be drawn between SLE presentation and increased risk for periodontitis. In furthering this study, the number of control patients enrolled should be increased in order to raise statistical power. By increasing either the entire sample size of test and control patients, or raising the controlto-case ratio, levels of significance may demonstrate that SLE patients are indeed at increased risk for deeper probing depths. For the purposes of the project, Mann-Whitney and Kruskal-Wallis non-parametric tests were applied to assess clinical data because of the relatively small sample sizes and presence of possible outliers. Younger patients that are completely edentulous or have aggressive forms of periodontitis would significantly affect the results of typical parametric analyses.

The healthy control patients were matched to the SLE group in terms of age and gender, as both factors have the potential to impact periodontitis risk and tooth loss. Systematic reviews 
of existing literature have shown males to be at greater risk for destructive periodontal disease [17] and that across all individuals, the prevalence of periodontitis increases with age [3]. By controlling these individual factors and creating similarity between test and control groups, the confounding effects of gender and age were minimized. However, there are other areas for potential confounding effects within the study worth mentioning. SLE patients are typically prescribed immunosuppressive, antimalarial, or steroidal drugs that may have some influence on periodontal parameters. A previous study by Mutlu [11] suggested that the anti-inflammatory medications taken by SLE patients were responsible for lower probing depths than control patients. Another unseen confounder could be the race or socioeconomic statuses of the subjects, which were not available in the patient records. Eke et al [18] reported that Black and Hispanic individuals had the highest prevalence of periodontitis in the United States. It is also established in periodontal literature that income and education levels are inversely related to periodontal disease susceptibility, due to disparities in oral hygiene behaviors and financial access to health care [19]. This information was not available in the patient records.

An interesting observation in the study was the significant difference in the secondary outcome of missing teeth. Compared to our healthy control population, the SLE patients had a significantly higher number of missing teeth, with two completely edentulous subjects. Considering that increased probing depths were not statistically dissimilar between test and control groups, there may be an alternative explanation to this observation. SLE patients frequently have symptoms of xerostomia and hyposalivation secondary to SLE that increase their susceptibility to dental caries and tooth loss. Physiologically, it has been theorized that inflammatory cell infiltrate from the autoimmune condition leads to fibrosis and degeneration of the salivary glands [20]. A study by Leite [21] demonstrated that $>75 \%$ of SLE patients suffered from hyposalivation, independent of the types of medications that they were taking. In 2018, Correa [22] performed a study on the oral-health related quality of life in SLE patients and found that SLE patients had an increased number of missing teeth, despite a higher frequency of tooth brushing relative to their healthy counterparts. The retrospective review of patient's dental histories did not provide information on the etiology of tooth loss, so it was not possible in the present study to comment on whether their edentulism was due to periodontal factors or dental decay.

Patients with greater degrees of edentulism naturally have fewer potential sites for periodontal breakdown, and this was accounted for in the statistical evaluation. Risk analysis on the Prevalence of increased periodontitis risk was based on the number of subjects with one or more sites with $\geq 5 \mathrm{~mm}$ probing depths, so completely edentulous patients were removed from our statistical analysis of prevalence. In contrast, the Extent of periodontitis risk revolved around the percentage of total sites remaining that were $\geq 5 \mathrm{~mm}$. Completely edentulous patients do not have any sites remaining and were not removed, as they did not have any statistical weight on the Extent of $\geq 5 \mathrm{~mm}$ probing depths. Lastly, all patients were included in the statistical analysis of missing teeth.

This was a cross-sectional study, and a cause-and-effect relationship could not be established between the periodontal parameters and SLE diagnosis. Previous studies that examined the relationship between autoimmune disorders and periodontitis can help explain the current findings. Meyer [10] suggested that long-standing autoimmune disorders like SLE do not alter immunologic parameters enough to produce significant periodontal pathology, whereas more acute conditions like leukemia can severely compromise the immune system and induce periodontal inflammation with subsequent bone loss. Conversely, other studies have demonstrated a shared pathogenesis between periodontitis and SLE. Both result in hyperactive B-cell responses, strong IgG antibody reactions, and accumulation of immune cells in affected tissues that can lead to exacerbation of the other condition [23].

Prospective studies in order to monitor SLE patients for periodontal breakdown over time would be valuable to our understanding between SLE and periodontal status. Notwithstanding the unknown etiology, results of the present study demonstrate SLE patients to be at-risk for increased tooth loss. It may not be unwarranted to propose early 
surveillance or maintenance protocols in order to mitigate any resulting pathologic effects on the oral condition.

\section{Conclusion}

Despite its limitations, the present study provides some further insight into the periodontitis risk in patients with SLE. The results support the conclusion that patients with SLE do not have an increased risk for periodontitis when compared to patients without SLE. Risk analysis on the prevalence and extent of deeper probing depths between SLE and control groups did not reach statistically significant p-values, indicating that the null hypothesis could not be rejected. Further studies with a larger sample size and elimination of unseen confounders are needed in order to validate the results. An interesting observation was the finding that SLE patients have a significantly greater number of missing teeth. The exact mechanism through which SLE patients experience periodontal breakdown and increased tooth loss is an avenue that merits future research.

\section{References}

1. Kumar RR, Jha S, Dhooria A, Dhir V (2019) Butterfly rash: hallmark of lupus. Qjm 112(11): 877.

2. Mak A, Tay SH (2014) Environmental factors, toxicants and systemic lupus erythematosus. Int J Mol Sci 15(9): 16043-16056.

3. Nazir MA (2017) Prevalence of periodontal disease, its association with systemic diseases and prevention. Int J Health Sci (Qassim) 11(2): $72-80$.

4. da Silva MK, de Carvalho ACG, Alves EHP, da Silva FRP, Pessoa LDS, et al. (2017). Genetic Factors and the Risk of Periodontitis Development: Findings from a Systematic Review Composed of 13 Studies of MetaAnalysis with 71,531 Participants. Int J Dent 2017: 1914073.

5. Rhodus NL, Johnson DK (1990) The prevalence of oral manifestations of systemic lupus erythematosus. Quintessence Int 21(6): 461-465.
6. Aurlene N, Manipal S, Prabu D, Rajmohan (2020) Prevalence of oral mucosal lesions, dental caries, and periodontal disease among patients with systemic lupus erythematosus in a teaching hospital in Chennai, Tamil Nadu. J Family Med Prim Care. 9(7): 3374-3380.

7. Fabbri C, Fuller R, Bonfá E, Guedes LK, D'Alleva PS, et al. (2014). Periodontitis treatment improves systemic lupus erythematosus response to immunosuppressive therapy. Clin Rheumatol 33(4): 505-509.

8. Bae SC, Lee YH (2020). Causal association between periodontitis and risk of rheumatoid arthritis and systemic lupus erythematosus: a Mendelian randomization. Z Rheumatol 79(9): 929-936.

9. Pessoa L, Aleti G, Choudhury S, Nguyen D, Yaskell T, et al. (2019) Host-Microbial Interactions in Systemic Lupus Erythematosus and Periodontitis. Front Immunol 10: 2602.

10. Meyer U, Kleinheinz J, Handschel J, KruseLösler B, Weingart D, et al. (2000) Oral findings in three different groups of immunocompromised patients. J Oral Pathol Med 29(4): 153-158.

11. Mutlu S, Richards A, Maddison P, Scully C. (1993) Gingival and periodontal health in systemic lupus erythematosus. Community Dent Oral Epidemiol 21(3): 158-161.

12. Calderaro DC, Ferreira GA, Corrêa JD, Mendonça SM, Silva TA, et al. (2017) Is chronic periodontitis premature in systemic lupus erythematosus patients? Clin Rheumatol. 36(3): 713-718.

13. Matuliene G, Pjetursson BE, Salvi GE, Schmidlin K, Brägger U, et al. (2008) Influence of residual pockets on progression of periodontitis and tooth loss: results after 11 years of maintenance. J Clin Periodontol. 35(8): 685-695. 
14. Waerhaug J (1978). Healing of the dentoepithelial junction following subgingival plaque control. II: As observed on extracted teeth. J Periodontol. 49(3): 119-134.

15. Lang NP, Tonetti MS (2003) Periodontal risk assessment (PRA) for patients in supportive periodontal therapy (SPT). Oral Health Prev Dent. 1(1): 7-16.

16. Kobayashi $\mathrm{T}$, Ito $\mathrm{S}$, Yasuda $\mathrm{K}$, Kuroda $\mathrm{T}$, Yamamoto K, et al. (2007) The combined genotypes of stimulatory and inhibitory $\mathrm{Fc}$ gamma receptors associated with systemic lupus erythematosus and periodontitis in Japanese adults. J Periodontol. 78(3): 467-474.

17. Shiau H., Reynolds MA (2010) Sex differences in destructive periodontal disease: a systematic review. J Periodontol. 81(10): 1379-1389.

18. Eke PI, Dye BA, Wei L, Slade GD, ThorntonEvans GO, et al. (2015) Update on Prevalence of Periodontitis in Adults in the United States: NHANES 2009 to 2012. J Periodontol. 86(5): 611-622.

19. Borrell LN, Crawford ND (2008) Social disparities in periodontitis among United States adults 1999-2004. Community Dent Oral Epidemiol. 36(5): 383-391.

20. Hammoudeh M (2018) Oral Manifestations of Systemic Lupus Erythematosus Patients.

21. Leite CA, Galera MF, Espinosa MM, de Lima PR, Fernandes V, et al. (2015) Prevalence of hyposalivation in patients with systemic lupus erythematosus in a brazilian subpopulation. Int J Rheumatol. 2015: 730285.

22. Corrêa JD, Branco LGA, Calderaro DC, Mendonça SMS, Travassos DV, et al. (2018) Impact of systemic lupus erythematosus on oral health-related quality of life. Lupus. 27(2): 283289.

23. Marques CP, Maor Y, de Andrade MS, Rodrigues VP, Benatti BB (2016) Possible evidence of systemic lupus erythematosus and periodontal disease association mediated by Toll-like receptors 2 and 4. Clin Exp Immunol. 183(2): 187-192. 